\title{
Modelling Individual Globular Clusters
}

\author{
Douglas C. Heggie ${ }^{1}$ and Mirek Giersz ${ }^{2}$ \\ ${ }^{1}$ School of Mathematics and Maxwell Institute for Mathematical Sciences, University of \\ Edinburgh, Edinburgh, EH9 3JZ, UK \\ email: d.c.heggie@ed.ac.uk \\ ${ }^{2}$ Nicolaus Copernicus Astronomical Center, Polish Academy of Sciences, Warsaw, Poland \\ email: mig@camk.edu.pl
}

\begin{abstract}
Astronomers have constructed models of globular clusters for over 100 years. These models mainly fall into two categories: (i) static models, such as King's model and its variants, and (ii) evolutionary models. Most attention has been given to static models, which are used to estimate mass-to-light ratios and mass segregation, and to combine data from proper motions and radial velocities. Evolutionary models have been developed for a few objects using the gaseous model, the Fokker-Planck model, Monte Carlo models and $N$-body models. These models have had a significant role in the search for massive black holes in globular clusters, for example.

In this presentation the problems associated with these various techniques will be summarised, and then we shall describe new work with Giersz's Monte Carlo code, which has been enhanced recently to include the stellar evolution of single and binary stars. We describe in particular recent attempts to model the nearby globular cluster M4, including predictions on the spatial distribution of binary stars and their semi-major axis distribution, to illustrate the effects of about 12 Gyr of dynamical evolution. We also discuss work on an approximate way of predicting the "initial" conditions for such modelling.
\end{abstract}

Keywords. methods: numerical, globular clusters: general, globular clusters: individual (M4)

\section{Introduction}

\subsection{Some astrophysical questions}

There are many reasons for constructing a dynamical model of a globular cluster, but they fall into two broad categories. First there are problems that can be tackled by constructing static (equilibrium) models, such as

(a) Inferring the mass from the surface brightness profile, radial velocities, proper motions and mass functions. In this way one can estimate the total mass of stars below the observational limit, such as faint white dwarfs (e.g. Drukier et al. 1988)

(b) Inferring the global mass function from local mass functions (e.g. Richer et al. 2004): then one can address the question of whether this is the same for all clusters.

(c) Measuring cluster distances by comparison of radial velocities and proper motions: the model is used to correct for rotation, or to link the different locations and stellar components which are observed by the different techniques (e.g. van de Ven et al. 2006).

Then there is a second range of questions which require dynamic evolutionary models, i.e. questions such as

(a) Inferring the primordial mass function from local, present-day mass functions: the model is used to correct for preferential escape of low-mass stars (e.g. Baumgardt \& Makino 2003).

(b) Inferring primordial parameters of the binaries from their present-day statistical properties: primordial abundance, period distribution, etc (e.g. Kroupa et al. 2001) 
(c) Determining the effect of dynamics on the estimation of mass through the virial theorem, which is affected by mass segregation (Fleck et al. 2006)

Note that these last few references do not deal with specific objects (which is the focus of the rest of this review) but with general trends.

This paper begins with a review of the methods and observational constraints which have been used to construct models of individual globular clusters, often with a view to answering the above types of question. Then we focus on one particular method, the Monte Carlo method, and its application to the nearby globular cluster M4.

\subsection{Methods and constraints}

\subsubsection{The methods}

A number of techniques have been used to construct static models, to answer questions of the first type:

(a) Plummer's model (Plummer 1911)

(b) King's model (King 1966; Peterson \& King 1975)

(c) Anisotropic models (Michie \& Bodenheimer 1963)

(d) Multi-mass models (Gunn \& Griffin 1979; Pryor et al. 1986; Dubath et al. 1990)

(e) Non-parametric models (Gebhardt \& Fischer 1995)

(f) Schwarzschild's method (van de Ven et al. 2006)

(g) Jeans' equations (Leonard et al. 1992)

Even this list may not be exhaustive.

Much less work has been done on dynamical evolutionary models of specific globular clusters, but the methods include

(a) Gas/fluid models (Angeletti et al. 1980 [M3])

(b) Fokker-Planck models (Cohn and co-workers: Grabhorn et al. 1992 [N6624], Dull et al. 1997 [M15]; Drukier 1993, 1995 [N6397]; Phinney 1993 [M15])

(c) Monte Carlo models (Giersz \& Heggie 2003 [ $\omega$ Cen])

(d) $N$-body models

The last of these should really not be on this list. Though it should be the method of choice, it has not been used for the purposes which are the focus of this paper. An example is the modelling of M15 by Baumgardt et al. (2003), who constructed a small version which, when scaled up, corresponded approximately to the conditions expected for M15. The difficulty is that unscaled $N$-body models are practically limited to $N$ of order $10^{5}$ at present (e.g. Baumgardt \& Makino 2003; Hurley 2007), whereas the median for the globular clusters at the present time is about $5 \times 10^{5}$. Though it might be hoped that the gap would be bridged by the next generation of computers, it must be recognised that all globular clusters at the present day have lost substantial numbers of stars, and the primordial median must have been higher. Note that it has become possible only relatively recently to carry out full simulations of open star clusters. The initial mass of M67, for instance, is estimated at about $19000 M_{\odot}$, i.e. about 10 times its present mass, and this simulation, with a realistic complement of primordial binaries, took of order 1 month (Hurley et al. 2005).

\subsubsection{The observational constraints}

Whichever method is chosen to model a globular cluster, there are a number of observational constraints to be satisfied. In historical order of first use we have

(a) Surface brightness and/or star counts, starting with Von Zeipel (1908);

(b) Radial velocities, whether central averaged values (Illingworth 1976) or radial velocities of individual stars (Gunn \& Griffin 1979)

(c) Pulsar accelerations (Phinney 1993; Grabhorn 1993) 
(d) Deep luminosity/mass functions, starting essentially with the advent of studies by several authors using HST (1995)

(e) Accurate proper motions (van den Bosch et al. 2006). (We refer here to models built to satisfy constraints imposed by observations of internal proper motions, and not to the use of proper motions to establish membership.)

\subsubsection{The Monte Carlo Model}

This paper focuses on an application of the Monte Carlo code developed essentially by Giersz $(1998,2001,2006)$. In this approach we assume spherical symmetry and dynamic equilibrium, and characterise each star by its energy $E$ and angular momentum $J$. The code repeatedly alters $E$ and $J$ to mimic the effects of gravitational encounters, using the theory of relaxation. The same theory underpins Fokker-Planck codes, and the basic Monte Carlo code provides essentially a Monte Carlo solution of the Fokker-Planck equation.

The Monte Carlo code is rather suitable for the addition of a number of other process, chiefly:

(a) The galactic tidal field, which is treated as a cutoff

(b) Binaries, whose interactions are treated using cross sections (from Spitzer (1987) for interactions with single stars, and expressions based on Mikkola (1983, 1984a,b) for interactions between binaries)

(c) Stellar evolution of single stars (Hurley et al. 2000) and binary stars (Hurley et al. 2002).

Each of these requires some comment, though further details are given by Giersz \& Heggie in this volume, and in Sec. 4 below.

(a) There are significant differences between a tidal field and a tidal cutoff, as these lead to somewhat different scalings of the dissolution time with $N$ (Baumgardt 2001). We have attempted to mimic this with a mass-dependent lowering of the escape energy.

(b) The Monte Carlo code described here has no triples, and so hierarchical triples (which are a common product of binary-binary encounters) have to be bypassed. Furthermore, the use of cross sections hinders the inclusion of star-star collisions during 3and 4-body encounters. Finally, the cross sections are not well known for unequal masses.

(c) Stellar evolution is implemented via the McScatter interface (Heggie et al. 2006). Besides the stellar evolution packages of Hurley et al. (referenced above) it also interfaces to the stellar evolution package SeBa in starlab (Portegies Zwart \& Verbunt 1996). At present, however, the latter is limited to solar metallicity, which is unsuitable for the study of old globular clusters.

In view of the above approximations and uncertainties, the testing and calibration of the Monte Carlo code against the results of $N$-body models (in the regime of small enough $N$ ) is an essential safeguard. Such studies are described by Giersz \& Heggie in this volume.

\section{The globular cluster M4}

This, one of the very nearest known globular clusters, was selected at the meeting MODEST-5 (Hamilton, Canada, 2004) as a target for concerted observational and theoretical effort, but so far little theoretical work has been carried out. Table 1 summarises some data for this fascinating object.

The proximity of M4 makes deep observational study possible. (See, for example, the poster by Sommariva et al. in this volume). For theoretical purposes too it is well placed for study because its binary population appears to be modest, and its initial mass may 
Table 1. Properties of M4

\begin{tabular}{ll}
\hline Distance from sun & ${ }^{a} 1.72 \mathrm{kpc}$ \\
Distance from GC & $5.9 \mathrm{kpc}$ \\
Mass & ${ }^{a} 63000 M_{\odot}$ \\
Core radius & $0.53 \mathrm{pc}$ \\
Half-light radius & $2.3 \mathrm{pc}$ \\
Tidal radius & $21 \mathrm{pc}$ \\
Half-mass relaxation time $\left(R_{h}\right)$ & $660 \mathrm{Myr}$ \\
Binary fraction & ${ }^{a} 1-15 \%$ \\
{$[\mathrm{Fe} / \mathrm{H}]$} & -1.2 \\
Age & ${ }^{b} 12 \mathrm{Gyr}$ \\
$A_{V}$ & ${ }^{a} 1.33$ \\
\hline
\end{tabular}

References: All data are from the current version of the catalogue of Harris (1996), except ${ }^{a}$ Richer et al. (2004) (though this is not always the original reference for the quoted number) and ${ }^{b}$ Hansen et al. (2004).

not have been very high, as we shall see. One complication for the Monte Carlo code, however, is that the orbit appears to be very elliptical (Dinescu et al. 1999), whereas we must assume a steady tidal field.

Table 2 describes the initial conditions which we adopted for this exercise. The primary observational data which we attempted to fit were

(a) The surface brightness profile (Trager et al. 1995)

(b) The radial velocity dispersion profile (Peterson et al. 1995)

(c) The V-luminosity function (Richer et al. 2004, from which we considered the results for the innermost and outermost of their four annuli) though several other observational comparisons will be described below. We do not have a systematic way of arriving at a best choice of initial parameters, though a possible approach is described towards the end of this paper. We began with a scaled-up version of the models we developed for the old open cluster M67 (see Giersz \& Heggie in this volume), but found that a binary population of $f_{b}=50 \%$ tended to produce a model with too low a concentration. Reducing the binary concentration to 5 or $10 \%$ produced a satisfactory surface brightness profile, but was somewhat too massive, because of an excess of low-mass stars, corresponding to a poor fit with the luminosity function. According to Baumgardt \& Makino (2003) it might be possible to correct this by devising a model which lost mass at a higher rate, but instead we elected to change the slope of the low-mass IMF from the canonical value of $\alpha=1.3$ (Kroupa 2007) to $\alpha=0.9$. (There is some justification for a lower value for low-metallicity populations.) By some experimentation we arrived at a model which gave a fair fit to all three kinds of observational data; see Table 3, and Figs. 1-4. Much of the disagreement in the total luminosity is due to our assumed distance to the cluster, which is significantly smaller than the value of $2.2 \mathrm{kpc}$ given by Harris (1996), though our surface brightness profile is also a little faint on average. The disagreement in the inner luminosity function at faint magnitudes may be attributable to the fact that the theoretical result assumes $100 \%$ completeness, while the observational data are uncorrected for completeness. A typical plot of a completeness correction is given by Hansen et al. (2002, Fig. 3).

It is worth noting that no arbitrary normalisation has been applied in these comparisons between our model and the observations. The surface brightness profile, for example, is computed directly from the $\mathrm{V}$-magnitudes of the stars in the Monte Carlo simulation.

Fig. 5 shows the colour-magnitude diagram of the model. This is of interest, not so much for comparison with observations, but for the presence of a number of interesting 
Table 2. Initial parameters for M4

\begin{tabular}{ll}
\hline Fixed parameters & \\
Structure & Plummer model \\
Stellar IMF & Kroupa double power law \\
Binary mass distribution & Kroupa et al. (1991) \\
Binary mass ratio & Uniform \\
Binary semi-major axis & Uniform in log, $2\left(R_{1}+R_{2}\right)$ to $50 \mathrm{AU}$ \\
Binary eccentricity & Thermal, with eigenevolution (Kroupa 1995) \\
Metallicity $Z$ & 0.002 \\
Age & $12 \mathrm{Gyr}$ \\
\hline Free parameters & \\
Mass & $M$ \\
Tidal radius & $r_{t}$ \\
Half-mass radius & $r_{h}$ \\
Binary fraction & $f_{b}$ \\
Slope of the lower mass function & $\alpha($ Kroupa $=1.3)$ \\
\hline
\end{tabular}

Table 3. Monte Carlo and King models for M4

\begin{tabular}{llll}
\hline Quantity & $\begin{array}{l}\text { MC model } \\
(t=0)\end{array}$ & $\begin{array}{l}\text { MC model } \\
(t=12 \mathrm{Gyr})\end{array}$ & $\begin{array}{l}\text { King model } \\
(\text { Richer } \text { et al. } 2004)\end{array}$ \\
\hline Mass $\left(M_{\odot}\right)$ & $3.40 \times 10^{5}$ & $4.61 \times 10^{4}$ & \\
Luminosity $\left(L_{\odot}\right)$ & $6.1 \times 10^{6}$ & $2.55 \times 10^{4}$ & $6.25 \times 10^{4}$ \\
Binary fraction $f_{b}$ & 0.07 & 0.057 & 0 \\
Low-mass MF slope $\alpha$ & 0.9 & 0.03 & 0.1 \\
Mass of white dwarfs $\left(M_{\odot}\right)$ & 0 & $1.81 \times 10^{4}$ & $3.25 \times 10^{4^{*}}$ \\
Mass of neutron stars $\left(M_{\odot}\right)$ & 0 & $3.24 \times 10^{3}$ & \\
Tidal radius $r_{t}(\mathrm{pc})$ & 35.0 & 18.0 & \\
Half-mass radius $r_{h}(\mathrm{pc})$ & 0.58 & 2.89 & \\
\hline
\end{tabular}

*: this is the quoted mass of "degenerates"

features. The division of the lower main sequence is simply an artifact of the way binary masses were selected (a total mass above $0.2 M_{\odot}$ and a component mass above $0.1 M_{\odot}$.) Of particular interest are the high numbers of merger remnants on the lower white dwarf sequence. There are very few blue stragglers. Partly this is a result of the low binary frequency, but it is also important to note that some formation channels are unrepresented in our models (in particular, collisions during triple or four-body interactions, though if a binary emerges from an interaction with appropriate parameters, it will be treated as merged.) These numbers also depend on the assumed initial distribution of semi-major axis, which is not yet well constrained by observations in globular clusters.

Photometric binaries are visible in Fig. 5, and these are compared with observations in the inner field of Richer et al. (2004) in Fig. 6. In this figure, the model histogram has been normalised to the same total number of stars as the observational one. We made no attempt to simulate photometric errors, but the bins around abscissa $=-0.75$ suggest that the binary fractions in the model and the observations are comparable. Fig. 7 shows that binaries have evolved dynamically as well as through their internal evolution. In particular the softest pairs been almost destroyed.

By 12 Gyr the binaries exhibit segregation towards the centre of the cluster, but perhaps in more subtle ways than might be expected (Figs. 8,9). When all binaries are considered, there is little segregation relative to the other objects in the system. (Most binaries in our model are of low mass.) But if one restricts attention to bright binaries, 


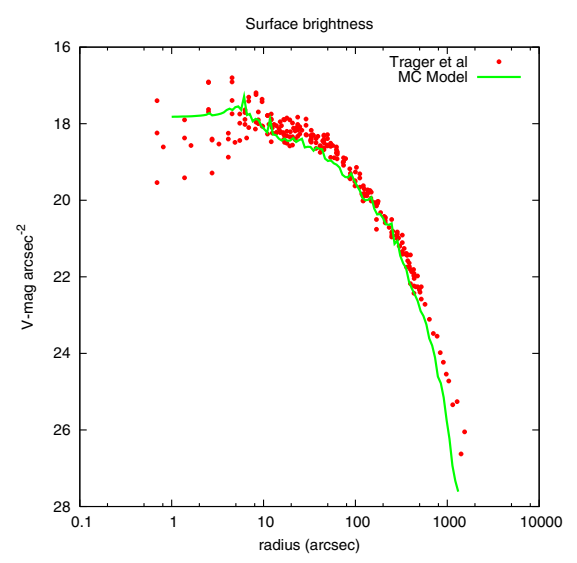

Figure 1. Surface brightness profile of our Monte Carlo model, compared with the data of Trager et al. (1995).

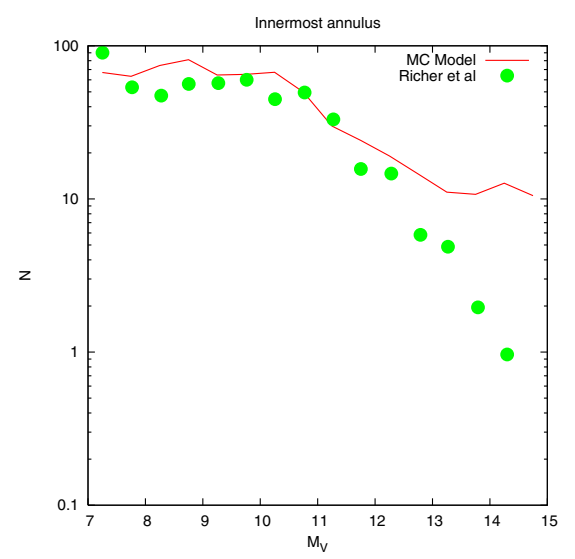

Figure 3. Luminosity function of our Monte Carlo model at the median radius of the innermost annulus in Richer et al. (2004), compared with their data.

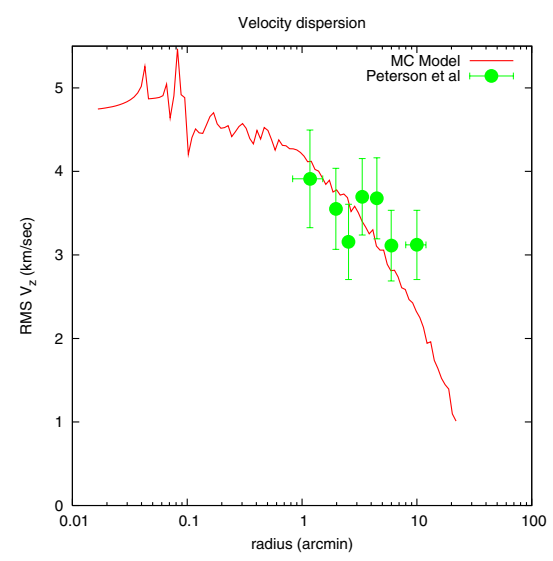

Figure 2. Velocity dispersion profile of our Monte Carlo model, compared with the data of Peterson et al. (1995).

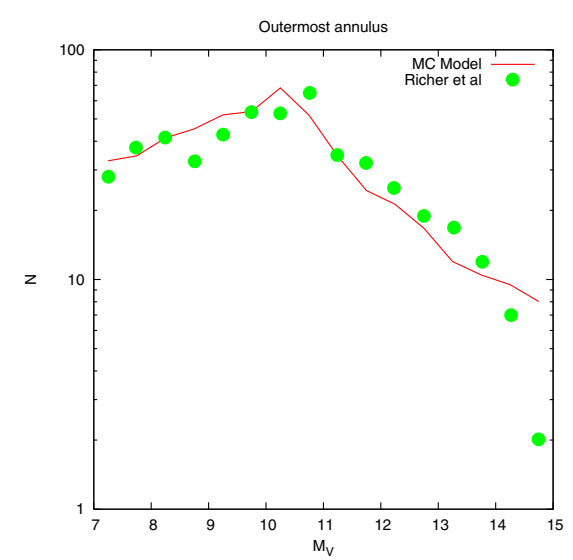

Figure 4. Luminosity function of our Monte Carlo model at the median radius of the outermost annulus in Richer et al. (2004), compared with their data.

which we here take to mean those with $M_{V}<7$ (i.e. brighter than about two magnitudes below turnoff), the segregation is very noticeable (Fig. 9), with a half-mass radius smaller by almost a factor of 2 than for bright single stars. Still, bright binaries are not nearly as mass-segregated as neutron stars (Fig. 8), which, incidentally, receive no natal kicks in our model.

These data do not reveal one very interesting feature of our model, which is that it exhibited core collapse at about 8 Gyr. Subsequently its core radius is presumably sustained by binary burning. Even non-primordial binaries may be playing a role here. To the best of our knowledge it has not previously been suggested that M4, which is classified as a "King" cluster by Trager et al. (1993), is a post-collapse cluster. This raises the long-dormant question of how it is possible for some clusters to exhibit collapsed cores if they also come with significant populations of primordial binaries. 


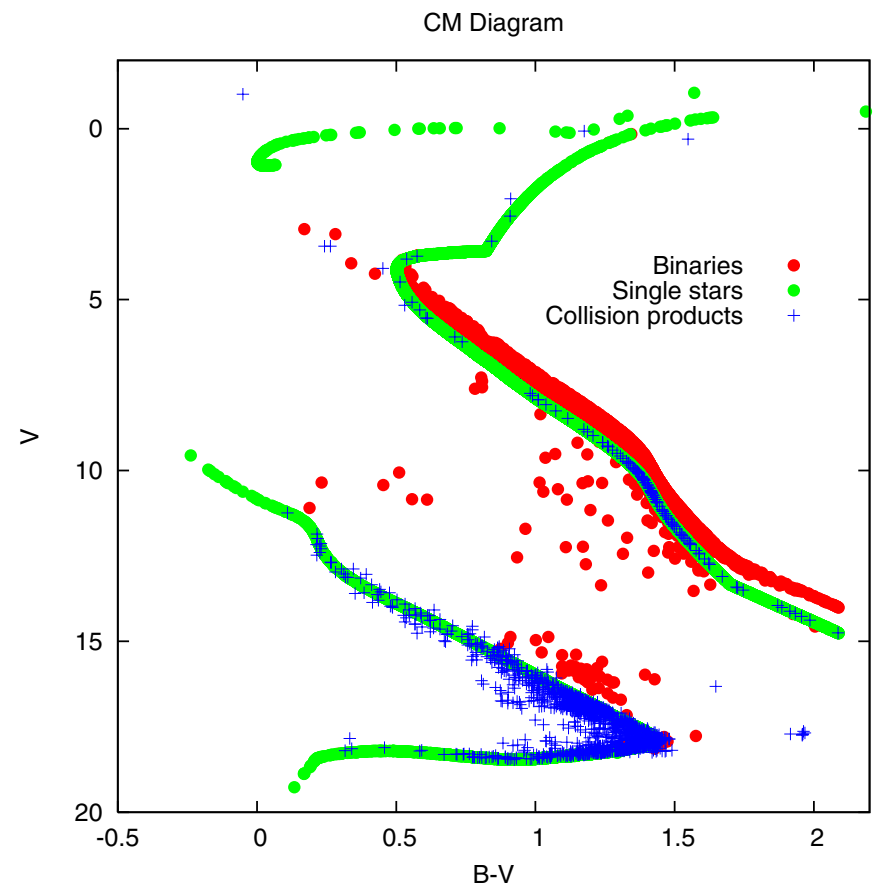

Figure 5. The colour-magnitude diagram at 12 Gyr. Green: single stars; red: binaries; blue pluses: collision or merger remnants.

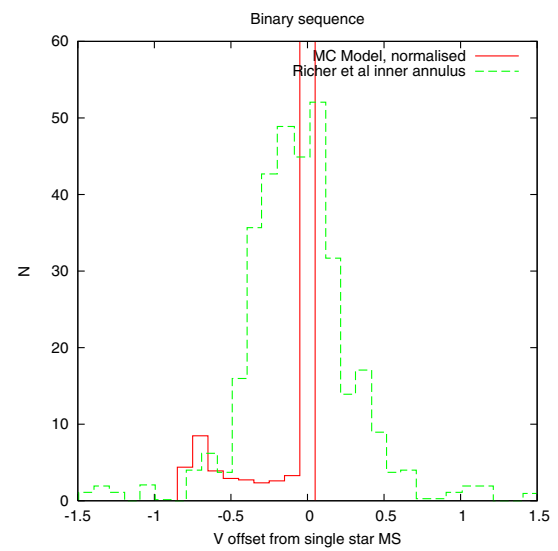

Figure 6. Histogram of $V$-offset from the main sequence, compared with the corresponding data from the innermost annulus studied by Richer et al. See text for details.

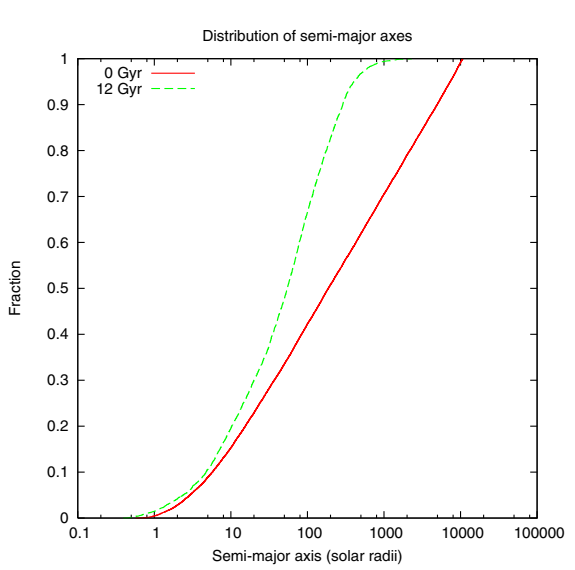

Figure 7. Distribution function of the semi-major axes of the binaries at $0 \mathrm{Gyr}$ and $12 \mathrm{Gyr}$. Units: solar radii.

\section{The search for initial conditions}

Each Monte Carlo model for this cluster takes a few days. Therefore the problem of finding appropriate initial conditions is a significant one. One needs a good starting guess, and then a rapid method for iterative improvement. Our techniques for dealing with these issues are still primitive, but have evolved in the course of this research. At the iterative stage we have employed scaled-up small models (with as few as $10^{4}$ objects sometimes), which are designed to relax at the same rate as a full-scale model. This requires a scaling 


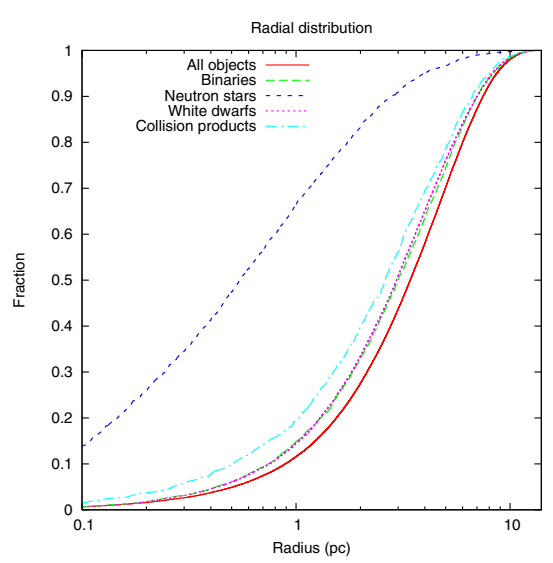

Figure 8. Radial distribution functions at 12 Gyr. Units: parsecs. The key identifies the class of object included. The distributions of white dwarfs and binaries are almost identical.

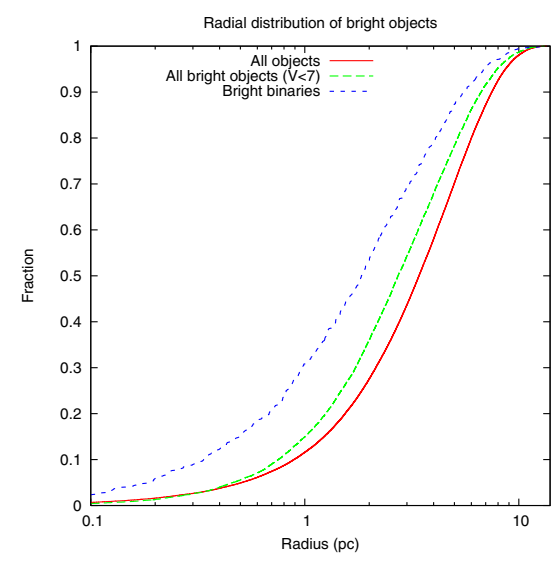

Figure 9. Radial distribution functions at 12 Gyr, showing the extent of segregation between bright single and bright binary stars $\left(M_{V}<7\right)$. Also shown for comparison is the distribution for all objects, as in Fig. 8. Units: parsecs.

of the length-scale, which does violence to binary interactions, but nevertheless it has been very useful. In this section we focus on the issue of finding starting values.

Consider first the problem of forwards evolution. Useful formulae for $M(t)$ are given by Lamers et al. (2005), which we have generalised to other IMF's and metallicities. To this we have added formulae for $r_{h}(t)$, based on very simple notions of adiabatic expansion (in response to mass-loss from stellar evolution) and tidal truncation. For the evolution of the core we have fitted simple expressions to the results on the time of core collapse given by Baumgardt \& Makino (2003), extended by new $N$-body simulations to a wider range of initial concentrations. Very simple expressions for $r_{c}(t)$, consistent with the initial and final values, can then be employed as a first approximation. Finally we drew from Baumgardt \& Makino a relation between $M$ and $\alpha$ (the slope of the lower mass function.) Putting these relations together, we have constructed a tool which we refer to as Quick Cluster Evolution, following S. Portegies Zwart. To apply this to generate initial conditions for our simulations, we can run QCE iteratively in reverse.

Further development of this tool should include the addition of binary heating, which certainly influences the evolution of the half-mass radius unless the primordial binary fraction is low enough, and more concentrated initial conditions than the King models to which we have restricted QCE so far.

\section{Discussion}

It is shown by Giersz \& Heggie (this volume) that Monte Carlo models can provide similar results to $N$-body models, in the range where comparison is possible, with similar physics (binaries, stellar evolution, etc.), except for a number of restrictions:

(a) Use of a tidal cutoff, instead of the tidal field. Though Sec. 1.2.3 summarises our current approach to this problem, other treatments are possible, and worth trying.

(b) Use of a static tide. The effects of tidal shocks have been studied by a number of authors (e.g. Kundic \& Ostriker 1995), and it would be possible to add the effects as another process altering the energies and angular momenta of the stars in the simulation. 
(c) Rotation: it has been shown (Kim et al. 2004) that, to the extent that rotating and non-rotating models can be compared, rotation somewhat accelerates the rate of core collapse. Rotation is hard to implement in this Monte Carlo model, however.

(d) Use of cross sections for triple/quad interactions: this limitation could be overcome by direct integration of the interactions, as is done by Fregeau \& Rasio (2007) in their version of the Monte Carlo scheme. It will be important to do so, as it would remove the dependence on cross sections which are not well known for unequal masses, and would also permit us to include the collisions between stars which commonly occur in long-lived few-body interactions (Hut \& Inagaki 1985).

(e) Neglect of triples: these are also commonly produced in binary-binary encounters (Mikkola 1984a), and it is desirable to include these as a third species (beyond single and binary stars). Their observable effects may be small, but of course there is one intriguing example in the very cluster we have focused on here (Thorsett et al. 1993).

Despite these limitations, not all of which are easily curable, Monte Carlo models are feasible in reasonable time for globular clusters, which are too large for direct $N$-body models. They yield predictions for mass segregation, luminosity functions, distributions of binary parameters, anisotropy, and many other kinds of data, which can hardly be obtained in any other way. (The only comparable method of which we are aware is the hybrid code of Giersz \& Spurzem 2003.) Even when $N$-body simulations eventually become possible, Monte Carlo models will remain as a quicker way of exploring the main issues, just as King models have continued to dominate the field of star cluster modelling even when more advanced methods (e.g. Fokker-Planck models) have become available.

In addition to some of the possible improvements mentioned above, it is our intention to extend the approach to a number of other objects, including a "collapsed-core" cluster such as NGC6624. We welcome all suggestions for observational or theoretical comparisons, either on M4 or on other objects.

\section{Acknowledgements}

J. Hurley's help has been invaluable, especially in regard to stellar and binary evolution, and he went to much trouble to assist us. K. Meyer has been largely responsible for developing QCE, following initial work with S. Portegies Zwart. Her work was funded by the Carnegie Trust for the Universities of Scotland. We thank H.B. Richer for comments on a previous draft.

\section{References}

Angeletti, L., Dolcetta, R., \& Giannone, P. 1980, Astrophys. Sp. Sci., 69, 45

Baumgardt, H. 2001, MNRAS, 325, 1323

Baumgardt, H., Hut, P., Makino, J., McMillan, S., \& Portegies Zwart, S. 2003, ApJL, 582, L21

Baumgardt, H. \& Makino, J. 2003, MNRAS, 340, 227

Dinescu, D. I., Girard, T. M., \& van Altena, W. F. 1999, AJ, 117, 1792

Drukier, G. A. 1993, MNRAS, 265, 773

Drukier, G. A. 1995, ApJS, 100, 347

Drukier, G. A., Fahlman, G. G., Richer, H. B., \& Vandenberg, D. A. 1988, AJ, 95, 1415

Dubath, P., Meylan, G., Mayor, M., \& Magain, P. 1990, A\&A, 239, 142

Dull, J. D., Cohn, H. N., Lugger, P. M., Murphy, B. W., Seitzer, P. O., Callanan, P. J., Rutten, R. G. M., \& Charles, P. A. 1997, ApJ, 481, 267

Fleck, J.-J., Boily, C. M., Lançon, A., \& Deiters, S. 2006, MNRAS, 369, 1392

Fregeau, J. M. \& Rasio, F. A. 2007, ApJ, 658, 1047

Gebhardt, K., \& Fischer, P. 1995, AJ, 109, 209

Giersz, M. 1998, MNRAS, 298, 1239 
Giersz, M. 2001, MNRAS, 324, 218

Giersz, M. 2006, MNRAS, 371, 484

Giersz, M. \& Heggie, D. C. 2003, MNRAS, 339, 486

Giersz, M. \& Spurzem, R. 2003, MNRAS, 343, 781

Grabhorn, R. P. 1993, Ph.D. Thesis, Indiana University

Grabhorn, R. P., Cohn, H. N., Lugger, P. M., \& Murphy, B. W. 1992, ApJ, 392, 86

Gunn, J. E. \& Griffin, R. F. 1979, AJ, 84, 752

Hansen, B. M. S., et al. 2002, ApJL, 574, L155

Hansen, B. M. S., et al. 2004, ApJS, 155, 551

Harris, W. E. 1996, AJ, 112, 1487

Heggie, D. C., Portegies Zwart, S., \& Hurley, J. R. 2006, New Astronomy, 12, 20

Hurley, J. R., Pols, O. R., \& Tout, C. A. 2000, MNRAS, 315, 543

Hurley, J. R., Tout, C. A., \& Pols, O. R. 2002, MNRAS, 329, 897

Hurley, J. R., Pols, O. R., Aarseth, S. J., \& Tout, C. A. 2005, MNRAS, 363, 293

Hurley, J. R. 2007, MNRAS, 379, 93

Hut, P. \& Inagaki, S. 1985, ApJ, 298, 502

Illingworth, G. 1976, ApJ, 204, 73

Kim, E., Lee, H. M., \& Spurzem, R. 2004, MNRAS, 351, 220

King, I. R. 1966, $A J, 71,64$

Kroupa, P. 1995, MNRAS, 277, 1507

Kroupa, P. 2007, ArXiv Astrophysics e-prints, arXiv:astro-ph/0703124

Kroupa, P., Gilmore, G., \& Tout, C. A. 1991, MNRAS, 251, 293

Kroupa, P., Aarseth, S., \& Hurley, J. 2001, MNRAS, 321, 699

Kundic, T. \& Ostriker, J. P. 1995, ApJ, 438, 702

Lamers, H. J. G. L. M., Gieles, M., Bastian, N., Baumgardt, H., Kharchenko, N. V., \& Portegies Zwart, S. 2005, $A \mathscr{E} A, 441,117$

Leonard, P. J. T., Richer, H. B., \& Fahlman, G. G. 1992, AJ, 104, 2104

Michie, R. W. \& Bodenheimer, P. H. 1963, MNRAS, 126, 269

Mikkola, S. 1983, MNRAS, 205, 733

Mikkola, S. 1984a, MNRAS, 207, 115

Mikkola, S. 1984b, MNRAS, 208, 75

Peterson, C. J. \& King, I. R. 1975, AJ, 80, 427

Peterson, R. C., Rees, R. F., \& Cudworth, K. M. 1995, ApJ, 443, 124

Phinney, E. S. 1993, in Djorgovski, S. G., Meylan G., eds, Structure and Dynamics of Globular Clusters, ASPCS 50, 141

Plummer, H. C. 1911, MNRAS, 71, 460

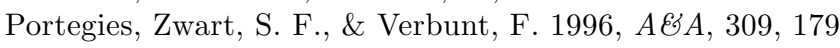

Pryor, C., Hartwick, F. D. A., McClure, R. D., Fletcher, J. M., \& Kormendy, J. 1986, AJ, 91, 546

Richer, H. B., et al. 2004, AJ, 127, 2771

Spitzer, L. 1987, Dynamical Evolution of Globular Clusters, Princeton, NJ, Princeton University Press, 1987

Thorsett, S. E., Arzoumanian, Z., \& Taylor, J. H. 1993, ApJL, 412, L33

Trager, S. C., Djorgovski, S., \& King, I. R. 1993, in Djorgovski, S.G., Meylan G., eds, Structure and Dynamics of Globular Clusters, ASPCS 50, 347

Trager, S. C., King, I. R., \& Djorgovski, S. 1995, AJ, 109, 218

van de Ven, G., van den Bosch, R. C. E., Verolme, E. K., \& de Zeeuw, P. T. 2006, A\&A, 445, 513

van den Bosch, R., de Zeeuw, T., Gebhardt, K., Noyola, E., \& van de Ven, G. 2006, ApJ, 641, 852

Von Zeipel, H. 1908, Annales de l'Observatoire de Paris, 25, 1 